Received: 05.11 .2018

Revised: 22.11 .2018

Accepted: 14.12 .2018

DOI: $10.17804 / 2410-9908.2018 .6 .083-089$

\title{
TEORETICAL BASES OF MAGNETOOPTICAL REFRACTOMETRY FOR TRANSPARENT MEDIA
}

\author{
A. V. Druzhinin ${ }^{1,2, a^{*}}$, A. P. Vladimirov ${ }^{1,3, b)}$, and E. V. Tsvetkova ${ }^{1, c)}$ \\ ${ }^{1}$ B. N. Yeltsin Ural Federal University, 19 Mira St, Ekaterinburg, 620002, , Russian Federation \\ ${ }^{2}$ IMP, Ural Branch of the Russian Academy of Sciences, \\ 18 S. Kovalevskoy St, Ekaterinburg, 620108, Russian Federation \\ ${ }^{3}$ IES, Ural Branch of the Russian Academy of Sciences, \\ 34 Komsomolskaya St, Ekaterinburg, 620049, , Russian Federation \\ a) (ID https://orcid.org/0000-0001-9944-096X ; druzhinin@imp.uran.ru; \\ b) (iD https://orcid.org/0000-0003-1653-0738; vap52@bk.ru; \\ c) $\otimes$ esenin1412@gmail.com \\ *Corresponding author. E-mail: druzhinin@imp.uran.ru \\ Address for correspondence: ul. S. Kovalevskoy, 18, 620108, Ekaterinburg, Russian Federation \\ Tel.: +7 (343) 3783872
}

This article discusses the possibility of applying the odd magnetooptical effect in transmitted light on the two-layer ferromagnetic-film-transparent-substrate system in order to determine the refractive index of the substrate. This phenomenon is also known as the Mayevsky-Druzhinin effect (MDE). The article provides a brief description of the MDE, in particular, its peculiarity in the angular dependence - the sign change at the Brewster angle of the substrate. The traditional method of angle determination is inferior in a number of characteristics to the one proposed in this paper, and this makes the MDE method promising for use in refractometry.

Keywords: refractometry, magnetooptics, equatorial effect, Brewster angle.

\section{Acknowledgment}

The work was performed within the state assignment from the Russian Ministry of Education and Science on the subject of Spin, No. AAAA-A18-118020290104-2.

\section{References}

1. GOST SSSR 28869-90. Materialy Opticheskie. Metody izmereniy pokazatelya prelomleniya [Optical materials. Methods of measuring the refractive index]. Moscow, IPK Izd-vo Standartov Publ., 1991. (In Russian).

2. Osipov V.V., Orlov A.N., Kashirin V.I., Lisenkov V.V. A setup for measuring the refractive index of a plane-parallel ceramic plate using the optical beam shift method. Instruments and Experimental Techniques, 2013, vol. 56, no. 1, pp. 80-83. DOI: 10.1134/S0020441213010107.

3. Maevsky V.M., Bolotin G.A. The theory of light passage through a magnetized film in view of weak spatial dispersion. The case of equatorial magnetization. Fizika Metallov i Metallovedenie, 1973, vol. 36, no. 2, pp. 11-21. (In Russian).

4. $\quad$ Druzhinin A.V., Lobov I.D., Maevsky V.M. Observation of the odd effect of light intensity variation as the light passes through an equatorially magnetized ferromagnetic film. Pismav ZhTF, 1981, vol. 7, no. 18, pp. 1100-1102. (In Russian). 
Подана в журнал: 05.11.2018

УДК 538.61

DOI: $10.17804 / 2410-9908.2018 .6 .083-089$

\title{
МЕТОДОЛОГИЧЕСКИЕ ОСНОВЫ МАГНИТООПТИЧЕСКОЙ РЕФРАКТОМЕТРИИ ПРОЗРАЧНЫХ СРЕД
}

\author{
А. В. Дружинин ${ }^{1,2,)^{*}}$, А. П. Владимиров ${ }^{1,3, \text { б) }}$, Е. В. Цветкова ${ }^{1, \text { в) }}$ \\ ${ }^{1}$ Уральский Федеральный Университет, пр. Мира, д. 19, Екатеринбург, Российская Федерация \\ ${ }^{2}$ Уральское отделение Российской Академии Наук, Институт физики металлов, \\ ул. С. Ковалевской, д. 18, Екатеринбург, Российская Федерация \\ ${ }^{3}$ Уральское отделение Российской Академии Наук, Институт машиноведения, \\ ул. Комсомольская, д. 34, Екатеринбург, Российская Федерация \\ а) ID https://orcid.org/0000-0001-9944-096X ; ه druzhinin@imp.uran.ru;
б) iD $\frac{\text { https://orcid.org/0000-0003-1653-0738 } ; \text { vap52@bk.ru; }}{\text { в) } \otimes \text { esenin1412@gmail.com }}$ \\ *Ответственный автор. Электронная почта: druzhinin@imp.uran.ru \\ Адрес для переписки: ул. С. Ковалевской, д. 18, 620108, Екатеринбург, Российская Федерация \\ Тел.: +7 (343) 378-38-72
}

В статье обсуждается возможность применения нечетного магнитооптического эффекта в проходящем свете на двухслойной системе ферромагнетик-прозрачная подложка для определения показателя преломления последней. Это явление также известно как эффект Маевского-Дружинина (ЭМД). В статье приводится краткое описание ЭМД, в частности характерной особенности угловой зависимости величины эффекта - смены знака при угле Брюстера подложки. Традиционный метод нахождения этого угла уступает предлагаемому по ряду характеристик, что делает применение ЭМД перспективным для использования в рефрактометрии.

Ключевые слова: рефрактометрия, магнитооптика, экваториальный эффект, угол Брюстера.

\section{1. Введение}

Показатель преломления среды является характеристикой, чрезвычайно чувствительной как к химической чистоте вещества, так и к механическому состоянию структуры образца. Современные технологии получения бездефектных сверхчистых оптических материалов нуждаются в бесконтактном, точном и быстром определении показателя преломления.

В настоящее время существует много различных способов измерения показателя преломления прозрачных сред [1], однако ни один из них не является универсальным. В каждом конкретном случае требуется применение адекватной методики измерения, и использовать соответствующее оборудование. Рекордные по чувствительности рефрактометрические методики чрезвычайно сложны и дороги [2]. Кроме того, они накладывают жесткие ограничения на качество подготовки исследуемых образцов.

Предлагаемый ниже магнитооптический способ определения угла Брюстера $\varphi_{b}$, тангенс которого является показателем преломления, обладает рядом преимуществ перед традиционной процедурой нахождения этого угла. Основными достоинствами магнитооптического способа являются: повышенная точность измерения угла, возможность работы с тонкими диэлектрическими слоями и отсутствие жестких требований к коллимации оптического пучка, что особенно важно при спектральных измерениях. Способ относится к разряду нулевых методик. 


\section{2. Постановка задачи и методы решения}

Взятый за основу предлагаемого метода нечетный интенсивностный гироэлектрический экваториальный магнитооптический эффект ( $\delta_{p}^{t}$-эффект) был предсказан в работе [3] и обнаружен позднее [4].

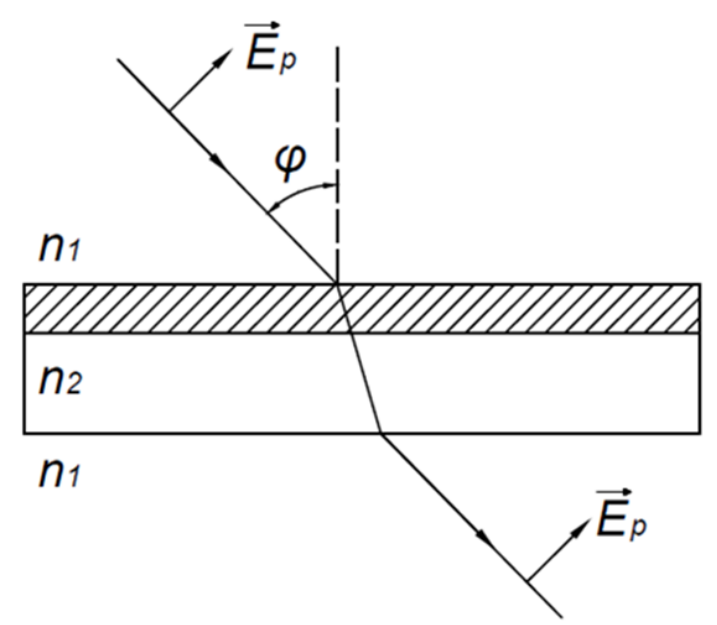

Рис. 1. Схема происхождения светового луча при наблюдении экваториального эффекта в проходящем свете

На рис. 1 изображен ход луча, проходящего через двухслойную систему полупрозрачной ферромагнитной пленки - прозрачную подложку. Магнитное поле, которое перемагничивает пленку, направлено перпендикулярно плоскости падения света (так называемая экваториальная геометрия). В случае, когда свет проходит сквозь полупрозрачную ферромагнитную пленку (пластинку) с одинаковыми внешними средами, эффект относительного приращения прошедшего $p$ - поляризованного света отсутствует. Это обусловлено тем, что экваториальные эффекты имеют граничную природу и в этом случае эффекты на обеих границах равны по величине и противоположны по знаку. Если же внешние среды по отношению к ферромагнетику обладают различными показателями преломления, полная компенсация нарушается. Поэтому этот эффект первоначально был назван разностным.

Этот эффект обозначают как $\delta_{p}^{t}$, где $\delta$ - относительное приращение интенсивности прошедшего через двухслойную среду света при перемагничивании образца. Индекс $\boldsymbol{p}$ обозначает поляризацию падающего света, а индекс $\boldsymbol{t}$ обозначает его пропускание через образец.

Зависимость величины эффекта от угла падения света определяется функцией [3]

$$
K_{p}(\varphi)=\left(n_{2}^{2} \cos \varphi-n_{1} \sqrt{n_{2}^{2}-n_{1}^{2} \sin ^{2} \varphi}\right) \sin \varphi \text {. }
$$

Данная функция (рис. 2 a) имеет немонотонный характер, изменяя свой знак при угле падения света, тангенс которого равен отношению показателей преломления внешних сред. Такой угол по определению - угол Брюстера. В случае, когда внешняя среда (вакуум или воздух) имеет $n_{1}=1$, мы получаем абсолютный показатель преломления подложки $n_{2}$.

Такая особенность угловой зависимости магнитооптического $\delta_{p}^{t}$-эффекта открывает возможность его использования для определения показателя преломления прозрачного вещества.

Традиционный способ определения угла Брюстера состоит в определении углового положения минимума (близкого к нулю) коэффициента отражения $R_{p}(\varphi) p$-поляризованного света (рис. 2 б) от плоского зеркально отполированного диэлектрического образца: 


$$
R_{p}(\varphi)=\frac{I}{I_{0}}
$$

где: $I_{0}-$ интенсивность падающего света; $I$ - интенсивность отраженного света.

Угловая зависимость этой величины определяется формулой Френеля:

$$
R_{p}(\varphi)=\left(\frac{n_{2}^{2} \cos \varphi-n_{1} \sqrt{n_{2}^{2}-n_{1}^{2} \sin ^{2} \varphi}}{n_{2}^{2} \cos \varphi+n_{1} \sqrt{n_{2}^{2}-n_{1}^{2} \sin ^{2} \varphi}}\right)^{2}
$$

В формуле (3) $n_{1}$ - показатель преломления внешней среды; $n_{2}-$ показатель преломления прозрачной среды; $\varphi$ - угол падения света.

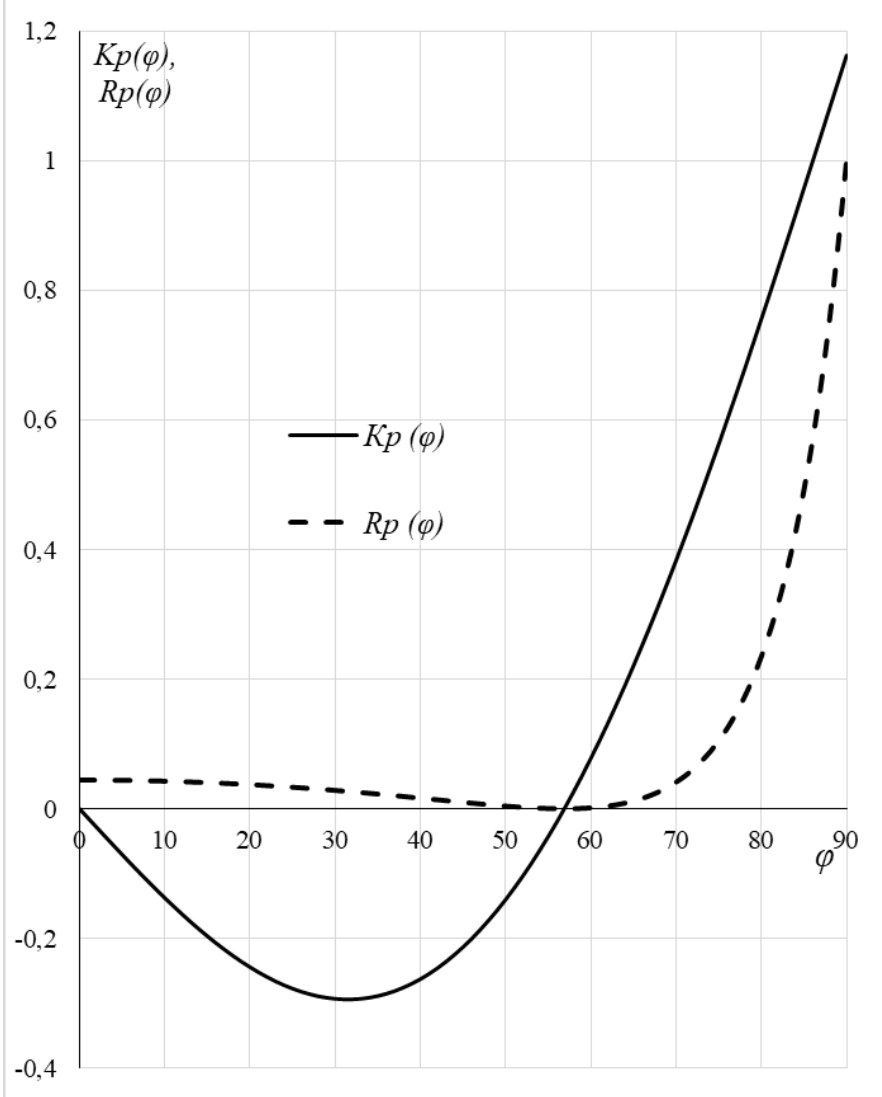

Рис. 2. Угловая зависимость: $a-$ функции $-K_{p}(\varphi)\left(n_{1}=1, n_{2}=1,5\right) ; \sigma$-коэффициента отражения $R_{p}$ для диэлектрика с $n=1,5$

На рис. 2 представлены обе угловые зависимости, имеющие зануление при угле Брюстера $\varphi_{B}$.

Свет с $p$-поляризацией, преломляясь, не отражается, т. е. он не различает границу раздела при угле падения, равном $\varphi_{5}$. Подобный эффект возникает в магнитооптическом эффекте для $p$-поляризованного света: противоположные границы ферромагнитного слоя дают один и тот же эффект приращения пропускания, но с противоположным знаком.

Традиционное определение угла Брюстера усложняется не только нулевыми значениями самого коэффициента отражения, но также равенством нулю его угловой производной, что накладывает сильные ограничение на коллимацию рабочего пучка света. К тому же повышение точности измерений на порядок требует уменьшение ошибки 
определения отношения $\Delta I / I$ на два порядка. Невысокая реальная точность этого метода $\Delta n \sim 10^{-2}-10^{-3}$; жесткие требования к сходимости светового луча и на толщину плоскопараллельного образца, когда невозможно исключить второй паразитный луч, сильно ограничивают возможности классического метода Брюстера.

В предлагаемом новом магнитооптическом методе ряд вышеперечисленных недостатков определения угла Брюстера отсутствуют.

Рассмотрим характерную угловую зависимость $-K_{p}(\varphi)$ (рис. $2 a$ ) для случая, когда показатель преломления внешней среды $n_{1}=1$, а показатель преломления подложки $n_{2}=1,5$. Точка перехода через ноль соответствует значению $\operatorname{arctg}\left(n_{2} / n_{1}\right)$, т. е. углу Брюстера материала подложки. В отличие от чисто оптического случая, угловая производная от эффекта равна не нулю, а величине

$$
-K_{p}^{\prime}(\varphi)=-\left(1-n_{2}^{2}\right)=1,25
$$

Поскольку поведение величины эффекта в окрестности его нуля близко к линейной зависимости, требования к сходимости зондирующего луча уменьшаются во много раз, а также упрощается процедура интерполяции.

\section{3. Результаты и обсуждение}

Обнаруженный в работе [4] линейный по намагниченности $\delta_{p}^{t}$ магнитооптический эффект в проходящем $p$-поляризованном свете (рис. 3) полностью подтвердил предсказание работы [3] по угловой зависимости эффекта. Граничный характер эффекта подтверждается инверсией его знака при обращении хода луча света.

Образец представлял собой двухслойную систему: полупрозрачную ферромагнитную пленку железа (10 нм), нанесенную на стеклянную подложку. При перемагничивании пленки в направлении, перпендикулярном плоскости падения света (экваториальная геометрия) прошедший свет, не изменяя своей поляризации, модулируется по амплитуде на частоте магнитного поля. Глубина такой модуляции достигает величины $1,5 \cdot 10^{-3}$. Это достаточно большой эффект, который легко измеряется. В зависимости от интенсивности прошедшего света, предельные (реально измеряемые с процентной ошибкой) эффекты относительного приращения интенсивности света достигают величины $1 \cdot 10^{-6}$.

Второй характерной угловой особенностью является поведение эффекта вблизи нормального падения света (рис. 4). Из графика следует, что значения эффекта по обе стороны от нулевого угла падения равны по величине и противоположны по знаку.

Такая особенность эффекта позволяет без использования процедуры автоколлимации определить значение нулевого (нормального) угла падения с большой точностью даже для сходящихся пучков. Нахождение второго нуля вблизи угла Брюстера для сходящегося пучка дает небольшую угловую ошибку с занижением величины угла из-за нелинейного характера функции $K_{p}(\varphi)$ (рис. 5). Однако знание вида этой функции позволяет провести интерполяционную процедуру для нахождения истинного угла Брюстера.

Расчет показывает, что абсолютная ошибка в определении показателя преломления магнитооптическим методом находится из выражения

$$
\Delta n_{2}=\frac{n_{2}^{2}+n_{1}^{2}}{\sqrt{\left(n_{2}^{2}-n_{1}^{2}\right)} \cdot \delta_{p}\left(\frac{\pi}{2}\right)} \cdot \frac{\Delta \mathrm{I}}{\mathrm{I}}
$$




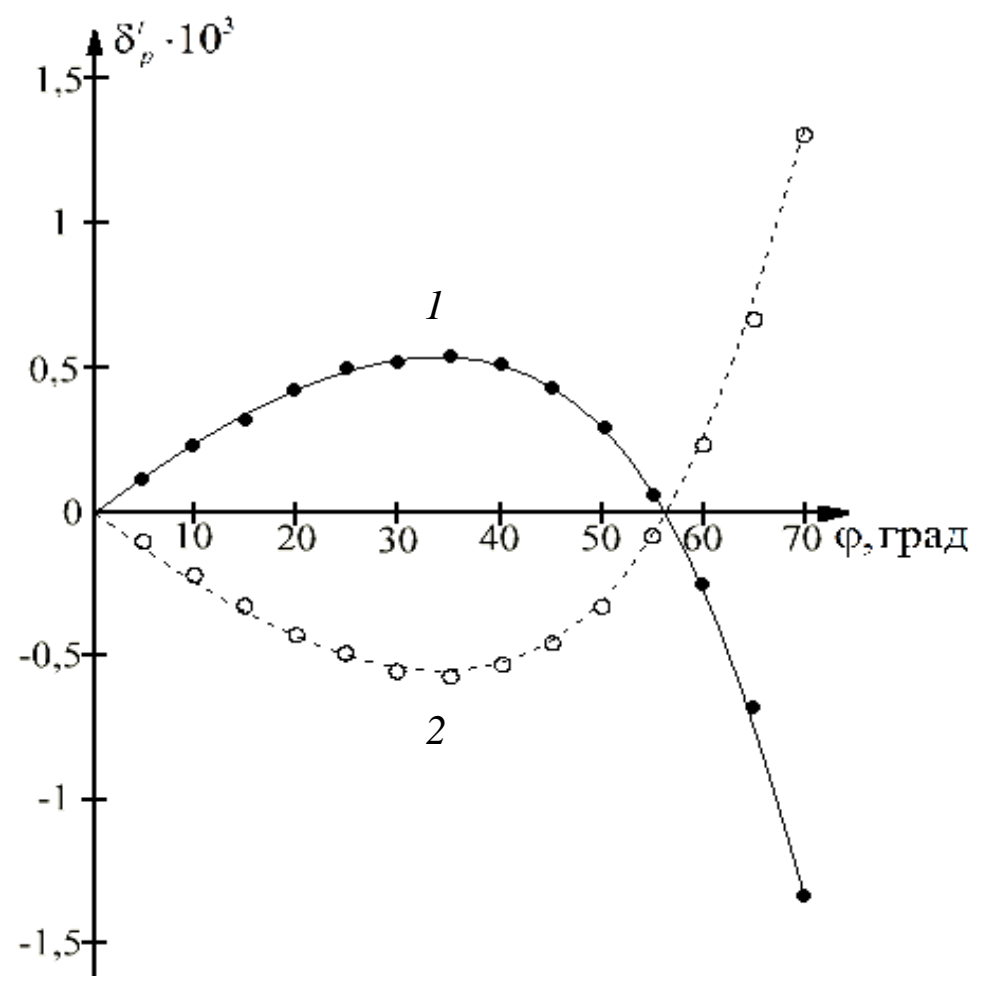

Рис. 3. Экспериментальная угловая зависимость $\delta_{p}^{t}$-эффекта: 1 - падение света со стороны пленки железа; 2 - падение света со стороны подложки [4]

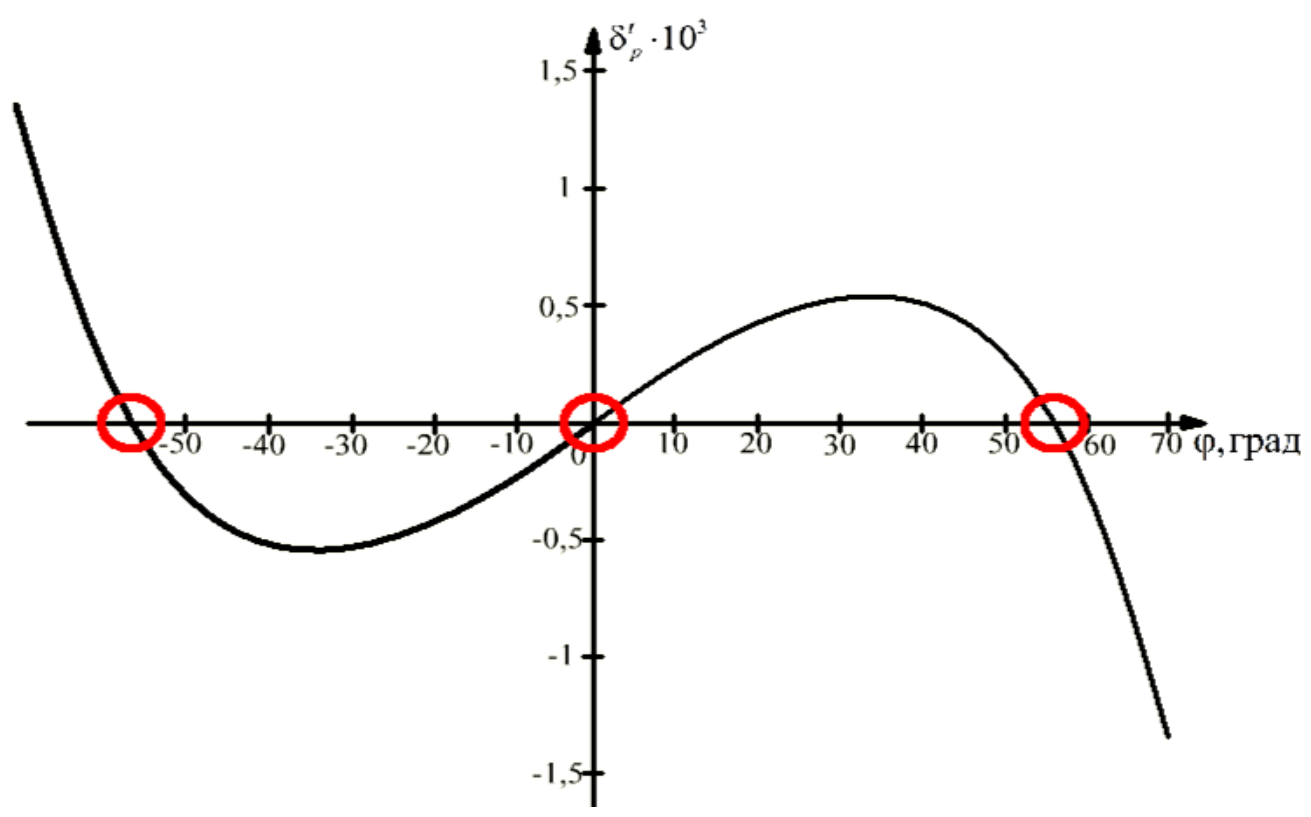

Рис. 4. Нулевые значения величины $\delta_{p}^{t}$-эффекта

Очевидно, эта ошибка тем меньше, чем больше значение магнитооптического эффекта при больших углах падения и чем меньше ошибка $\Delta I / I$ измерения переменной составляющей относительно величины проходящего светового потока. При современном уровне точности измерения интенсивностных эффектов $\Delta I / I \sim 1 \cdot 10^{-7}$, при значениях $\delta_{p}\left(\frac{\pi}{2}\right) \sim 2$. $10^{-3} ; n_{2} \sim 1,5 ; n_{1}=1$ формула (5) дает величину $\Delta n_{2} \simeq 0,6 \cdot 10^{-4}$.

Эта ошибка примерно на порядок меньше, чем при использовании эллипсометрического способа или классического метода угла Брюстера. 


\section{4. Заключение}

Представленный в работе магнитооптический метод для определения показателя преломления прозрачных сред, обладая простотой фотометрической процедуры и высокой точностью, имеет ряд преимуществ по сравнению с традиционным методом: возможность измерения тонких слоев, пленок в сходящихся пучках, что особенно важно для спектральных измерений. Качество ферромагнитной пленки, а также ее химический состав, для задачи рефрактометрии материала подложки не играет ни какой роли.

Предлагаемый магнитооптический способ определения показателя преломления прозрачных сред технически выполним и, обладая рядом преимуществ перед известными способами, может найти применение в рефрактометрических методах физики и химии твердого тела.

\section{Благодарность}

Работа выполнена в рамках государственного задания МИНОБРНАУКИ России (тема «Спин» №AAAA-A18-118020290104-2).

\section{Литература}

1. ГОСТ СССР 28869-90. Материалы оптические. Методы измерений показателя преломления. - Москва : ИПК Издательство стандартов, 1991.

2. A setup for measuring the refractive index of a plane-parallel ceramic plate using the optical beam shift method / V. V. Osipov, A. N. Orlov, V. I. Kashirin, V. V. Lisenkov // Instruments and Experimental Techniques. - 2013. - Vol. 56, no. 1. - P. 80-83. - DOI: 10.1134/S0020441213010107.

3. Маевский В. М., Болотин Г. А. К теории прохождения света через намагниченную плёнку при учёте слабой пространственной дисперсии. Случай экваториального намагничивания. - Физика металлов и металловедение. - 1973. - Т. 36, № 2. - С. 241-252.

4. Дружинин А. В., Лобов И. Д., Маевский В. М. Наблюдение нечётного эффекта изменения интенсивности света при его прохождении через экваториально намагниченную ферромагнитную плёнку // Письма в ЖТФ. - 1981. - Т. 7, вып. 18. - С. 1100-1102. 